\title{
La incidencia de la opinión social en el grado de vulnerabilidad de los edificios patrimoniales. El caso del centro histórico de Popayán (Colombia)
}

\author{
Ma Isabel Turbay Varona, Rocío Ortiz, María Arana, Pilar Ortiz
}

\begin{abstract}
Resumen: El patrimonio urbano y arquitectónico de muchas ciudades en Hispanoamérica es vulnerable frente a amenazas de diversa índole; la exposición a fenómenos naturales y antrópicos son muchas veces las causas de su destrucción, sin embargo, uno de los factores más importantes que sin duda tiende a debilitarlo, es su relación directa e indirecta con las sociedades comprometidas y por tanto, el valor que ellas han de asignarle; por este motivo, la opinión de los ciudadanos se convierte en un elemento constituyente de la preservación o destrucción del patrimonio histórico. Este trabajo se centra en analizar la opinión social de los habitantes de Popayán, Colombia, sobre el patrimonio arquitectónico de su centro histórico. El instrumento Las encuestas fueron diseñadas por un equipo interdisciplinario de arquitectos, ambientalistas, químicos, historiadores, matemáticos, ingenieros, ... que trabajan en Inteligencia Artificial aplicada a la conservación preventiva de edificios patrimoniales, y fueron revisadas por un sociólogo. Los resultados se analizaron como parte del insumo para la construcción de herramientas para determinar los factores involucrados en el grado de debilidad que sufren los edificios históricos y estimar la vulnerabilidad y peligrosidad, ante distintas amenazas.
\end{abstract}

Palabras clave: Conservación, grado de vulnerabilidad, opinión social, valor del patrimonio

\section{The incidence of social opinion on the degree of vulnerability of heritage buildings. The case of the historic center of Popayán (Colombia)}

\begin{abstract}
The urban and architectural heritage of many cities in Latin America is vulnerable to threats of various kinds; exposure to natural and anthropic phenomena are many times the causes of its destruction, however, one of the most important factors that undoubtedly tends to weaken it, is its direct and indirect relationship to committed societies and, therefore, the value that they assign it, because of that, the citizen opinion becomes a constituent element of cultural heritage preservation or destruction. This work focuses on analyzing the social opinion of the inhabitants of Popayan (Colombia), about the architectural heritage of the historical center. The surveys were designed by an interdisciplinary team of architects, environmentalists, chemists, historians, mathematics, engineering, ... that work on Artificial Intelligence applied to the preventive conservation of heritage buildings, and were revised by a sociologist. The results were analyzed as a tool to determine the factors involved in the degree of weakness that historical buildings suffer and estimate the vulnerability and hazards, facing different threats.
\end{abstract}

Keywords: Conservation, grade of vulnerability, social opinion, heritage value

\section{Introducción}

Para precisar el valor patrimonial de un bien es importante entender, el concepto de significación cultural, desde las recomendaciones de las cartas internacionales, como la carta de Burra (ICOMOS 1999), donde se especifica que los conjuntos de valores asignados a los monumentos, incluyen el valor estético, histórico, científico, social o espiritual que toman su forma en los lugares, en su materialidad, en el entorno, en el uso, en asociaciones, significados, registros, sitios relacionados y objetos relacionados. Por tanto la percepción acerca de estos valores, implica el reconocimiento y representatividad de las características y cualidades de los objetos, por parte de determinados grupos o individuos, que a su vez, establecen conexiones direccionadas a fortalecer, o no, la identidad comunitaria de los pueblos y que pueden emplearse también, como una función social en la cual una población, las toma, no solo como referente identitario, sino también como fuente potencial de desarrollo y relacionamiento social cotidiano (Llull Peñalba 2005).

Es necesario abordar también la relación entre el valor patrimonial y la conservación, lo cual se interpreta como 
un campo complejo (Manzini 2011), en el que puede ser determinante la evaluación de la opinión social. Explica Caraballo (Caraballo Perichi 2008) que alrededor de la significación acumulativa y en concordancia con la permanencia y evolución de los monumentos, se integra el contexto físico y temporal; mientras Garré (Garré 2001) establece que, el atributo (objeto) que representa ese valor, pudo en otro momento haber representado otros, incluso contradictorios, al que en el presente se le asigna; este proceso de re-significación resulta a partir de la evolución cultural de las comunidades que intervienen en este proceso y de su percepción acerca del patrimonio. Por tanto, el concepto de valor cultural tiene que ver directamente con el de la conservación del patrimonio, como describe García Canclini (García Canclini 1999). Es evidente que la interpretación social de los edificios y lugares puede tener diferentes matices en un rango de valor, según los grupos o individuos, y donde se involucran los intereses propios, cuestionados en diferentes entornos y estados, ocasionando la falta de comprensión del significado del patrimonio, asociada a la obsolescencia, abandono o pérdida de los monumentos (Manzini 2011) o por el contrario puede servir como instrumento de salvaguarda al patrimonio mismo y de reafirmación de la identidad en un conjunto social.

Las investigaciones dirigidas a identificar y medir los riesgos del patrimonio histórico son imprescindibles en la generación de insumos para la gestión de los entornos y los edificios históricos. En los avances en este campo, son la clave para garantizar la conservación, las propuestas de metodologías que identifiquen las debilidades del patrimonio, con el uso de herramientas que permitan la toma de decisiones.

La inclusión del valor social asociado a la vulnerabilidad no puede evaluarse solo en función de un listado de valores culturales implícitos a la normativa de los sitios. En la actualidad, las normativas de muchos países implican la catalogación de los monumentos, estos inventarios de valores intrínsecos reconocidos y protegidos, deben considerarse incompletos para la conservación del patrimonio; como se aborda en el estudio sobre la vulnerabilidad sísmica en Chile (Diaz Fuentes 2017), y es por tanto necesario involucrar la opinión de la sociedad, para otorgar mayor legitimidad hacia el valor que representa el patrimonio; de lo contrario, es más probable que aunque un bien esté reconocido desde el ámbito formal no cuente con la apropiación social suficiente y por tanto no se pueda garantizar su preservación en el futuro.

En los manuales de riesgo, la vulnerabilidad suele recogerse como la susceptibilidad de los bienes culturales ante una perturbación, es decir la debilidad inherente del patrimonio (UNESCO 1977) por tanto, la opinión de la sociedad no puede ser subvalorada ante la fragilidad de los monumentos, ya que puede ser reflejo de vitalidad o quebranto ante las diferentes amenazas. La importancia de este valor social del patrimonio se ha podido observar a nivel internacional tras el incendio de la Catedral de Notre Dame de París en 2019.

Este estudio complementa las metodologías propuestas por diversos autores (Ortiz y Ortiz 2016; Prieto et al. 2020; Turbay, Ortiz y Ortiz 2019) que tienen como objetivo calcular las vulnerabilidad en edificios históricos para minimizar los riesgos y la pérdida de elementos patrimoniales. Este tipo de investigaciones de evaluación de riesgos y estimación de la vulnerabilidad, incluyen estudios sistemáticos en los que se verifican las amenazas de tipo natural y antrópico, valorando el grado de afección mediante matrices de vulnerabilidad o inferencias calculadas por medio de inteligencia artificial; en los que se analizan un conjunto de variables. La opinión social del patrimonio histórico es una variable hasta ahora no incluida, que puede ser relevante como factor en la aproximación de escenarios de riesgo.

El modelo de evaluación de la opinión social que se aplicó en Popayán, tuvo como objetivo identificar la opinión de los ciudadanos como un factor que incide en la vulnerabilidad, y estimarlo como una nueva variable directamente proporcional a la debilidad o fortaleza del patrimonio ante las amenazas naturales o antrópicas. Este nuevo factor podría, a su vez, agregarse a las metodologías propuestas por varios autores (Ortiz y Ortiz 2016; Prieto et al. 2020; Turbay, et al. 2019) para la estimación de la vulnerabilidad de edificios históricos.

\section{Metodología}

La opinión social se ha evaluado mediante una encuesta de carácter anónimo, llevada a cabo en el casco histórico de la ciudad, específicamente en algunos puntos estratégicos del centro como son el Parque Caldas, uno de los lugares de mayor concurrencia, y en tres plazoletas de iglesias donde también se evidencia alto flujo peatonal diario (Alcaldía de Popayán 2015).

Las encuestas estuvieron dirigidas a ciudadanos colombianos o extranjeros, mayores de 18 años residentes en la ciudad, esto abarca a la población que ha establecido su domicilio de manera permanente en esa área y no incluye a la población que ejerce movilidad temporal o no definitiva, a través de movimientos diarios por distintas razones (trabajo, estudio, recreo, comercio, ...), y que puede implicar el traspaso de fronteras municipales como es el caso de Popayán, ya que es el principal centro urbano del departamento (Ministerio de Ambiente, Vivienda y desarrollo territorial 2004).

Para evaluar la muestra necesaria para que los resultados fuesen representativos, se tuvo en cuenta la población total estimada para Popayán (Colombia) en 2018, cercana a las 277.270 personas en la zona urbana, según los resultados del censo nacional de población y vivienda 
(DANE 2019); la muestra se segmentó por medio de las variables de sexo y edad de acuerdo a los datos de la pirámide poblacional. El total de encuestas fue de 384 , para obtener un $5 \%$ de error muestral y un $95 \%$ de confianza, de estas, 201 dirigidas a mujeres y 183 a hombres, de las cuales a su vez estuvieron distribuidos en 8 rangos de edad acorde con la densidad poblacional de cada grupo para la representatividad de la muestra.

Las encuestas en las que se basa este modelo han sido aplicadas en otras ciudades como Sevilla, Osuna y Marchena en España (Benítez et al. 2020). El proceso de entrevistas se realizaró entre noviembre y diciembre de 2018, de forma aleatoria entre los ciudadanos.

La primera parte de la encuesta reúne una caracterización social de los encuestados, que permitió conocer aspectos claves de los ciudadanos como la edad, el género, el lugar de origen, ocupación, tiempo de residencia en la ciudad y el nivel de estudios alcanzados, que posteriormente fueron relacionados con los resultados de las preguntas, que contenían las variables sobre la opinión social. La segunda parte de la encuesta [tabla I] permitió al ciudadano evaluar los ítems o afirmaciones para dar cuenta de su opinión sobre aspectos referentes a las problemáticas que sufren los monumentos, como el nivel de conservación, la participación ciudadana e institucional, así como la apropiación y conocimiento sobre el patrimonio edificado de Popayán. En todas las preguntas se puede valorar entre 1 a 5, siendo 1 muy poco importante para el entrevistado, y el valor 5 , implica que el concepto es considerado como muy importante por el entrevistado.
Los resultados de aplicación de las encuestas se ponderan mediante la opinión de los encuestados, para evidenciar el valor que representa el patrimonio, traducido en la preocupación de los ciudadanos hacia su conservación y su afectación en la vulnerabilidad del patrimonio de la ciudad.

El porcentaje de respuestas de cada valor (PRv), fue calculado con base al número total de respuestas obtenidas por cada uno de los valores (TRv) y dividida entre el sumatorio total de respuestas obtenidas por el valor correspondiente ( $\Sigma T R v)$ [Tabla II].

$$
\operatorname{PRv}(i)=\frac{\operatorname{TRv}(i)}{(\Sigma T R v)} * 100
$$

El promedio ponderado del grado de vulnerabilidad se obtiene, mediante el sumatorio de la multiplicación de cada porcentaje de respuestas de cada valor (PRv) por su Valor de la opinión social (V):

$$
\text { Grado de vulnerabilidad }=\sum\left[\operatorname{PRv}(\mathrm{i})^{*} \mathrm{~V}(\mathrm{i})\right]
$$

Esta valoración se encuentra en la escala entre 1 y 5 , siendo 1 en los casos donde la preocupación social por el patrimonio implicaría un patrimonio menos vulnerable, y 5 , en las ciudades en las que no hay preocupación social por el patrimonio, y esto hace que los monumentos sean más vulnerables.

Tabla I.- Resumen de la encuesta

\begin{tabular}{|l|c|c|c|c|c|}
\hline \multicolumn{1}{|c|}{ Preguntas } & $\begin{array}{c}\text { Muy poco } \\
\text { importante/ } \\
\text { Muy bajo }\end{array}$ & $\begin{array}{c}\text { Poco } \\
\text { importante/ } \\
\text { Bajo }\end{array}$ & $\begin{array}{c}\text { Algo } \\
\text { importante/ } \\
\text { Medio }\end{array}$ & $\begin{array}{c}\text { Importante/ } \\
\text { Alto }\end{array}$ & $\begin{array}{c}\text { Muy } \\
\text { importante/ } \\
\text { Muy alto }\end{array}$ \\
\hline $\begin{array}{l}\text { A. iEs importante conservar y mantener los } \\
\text { monumentos de mi ciudad? }\end{array}$ & 1 & 2 & 3 & 4 & 5 \\
\hline $\begin{array}{l}\text { B. ¿Considero que los monumentos de mi ciudad son } \\
\text { bien conocidos por sus habitantes? }\end{array}$ & 1 & 2 & 3 & 4 & 5 \\
\hline $\begin{array}{l}\text { C. iCreo que los políticos de mi ciudad se preocupan } \\
\text { por los monumentos? }\end{array}$ & 1 & 2 & 3 & 4 & 5 \\
\hline $\begin{array}{l}\text { D. ¿Realizo visitas frecuentes a museos, monumentos, } \\
\text { etc? }\end{array}$ & 1 & 2 & 3 & 4 & 5 \\
\hline $\begin{array}{l}\text { E. ¿Los monumentos tienen un papel importante a la } \\
\text { hora de planificar mis viajes? }\end{array}$ & 1 & 2 & 3 & 4 & 5 \\
\hline
\end{tabular}

\begin{tabular}{|c|c|c|c|c|c|c|}
\hline $\begin{array}{c}\text { Escala de valor de cada } \\
\text { pregunta }\end{array}$ & $\begin{array}{l}\text { Valor muy } \\
\text { bajo }\end{array}$ & Valor bajo & Valor medio & Valor alto & $\begin{array}{l}\text { Valor muy } \\
\text { alto }\end{array}$ & \\
\hline $\begin{array}{l}\text { Valor de la opinión social } \\
\text { (V) }\end{array}$ & 1 & 2 & 3 & 4 & 5 & \\
\hline $\begin{array}{l}\text { Total respuestas de cada } \\
\text { valor (TRv) }\end{array}$ & (TRv) 1 & $(\mathrm{TRv}) 2$ & $(T R v) 3$ & $(\mathrm{TRv}) 4$ & $(\mathrm{TRv}) 5$ & $\begin{array}{l}\text { Sumatorio total } \\
\text { respuestas }(\Sigma T R v)\end{array}$ \\
\hline
\end{tabular}

Tabla II.- Método para el cálculo de opiniones por escala de valor 


\section{Resultados y discusión}

\section{—Contexto social de la ciudad de Popayán}

Popayán está ubicada geográficamente en el Valle de Pubenza, entre la cordillera occidental y central al suroeste de Colombia, en el departamento del Cauca [figura 1], zona con alto riesgo sísmico. La vocación del departamento es rural, y esto se ve reflejado en las dinámicas y actividades de la población de composición cultural diversa, convirtiéndose la ciudad, en un centro de comercio donde interactúan los municipios cercanos. Popayán ha sido en las últimas décadas epicentro de migraciones del campo a la ciudad por desplazamientos forzados a causa del conflicto armado en Colombia. El turismo es uno de los motores económicos de la ciudad, que se activa especialmente en el mes de marzo y abril, cuando se celebra la Semana Santa, declarada Patrimonio Cultural inmaterial de la Humanidad.
La estructura productiva de la ciudad no está muy diversificada, y en el contexto social, funciona como un núcleo que acoge a un gran número de estudiantes foráneos al ser una atractiva opción para la realización de estudios superiores en diferentes universidades, por lo que su economía se apoya en actividades relacionadas. No obstante, hay una alta tasa de desempleo respecto a otras ciudades del país, así como los trabajos informales que no están amparados bajo las garantías legales para los trabajadores. El centro histórico de la ciudad actúa como centro político-administrativo, donde funcionan las instituciones gubernamentales, bancarias y sitios de uso comercial. Se trata del centro histórico más grande de Colombia, y pese a los grandes avatares sufridos por los sismos, mantiene su imagen de ciudad colonial, considerado Bien de Interés Cultural de ámbito nacional, por el valor histórico artístico de sus edificaciones [figura 2].

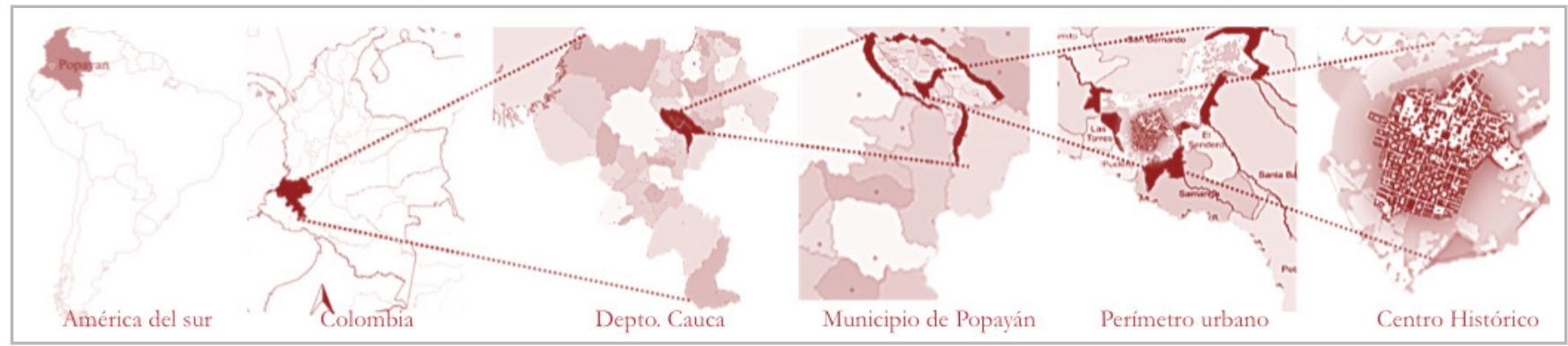

Figura 1.- Ubicación del Centro Histórico de Popayán.

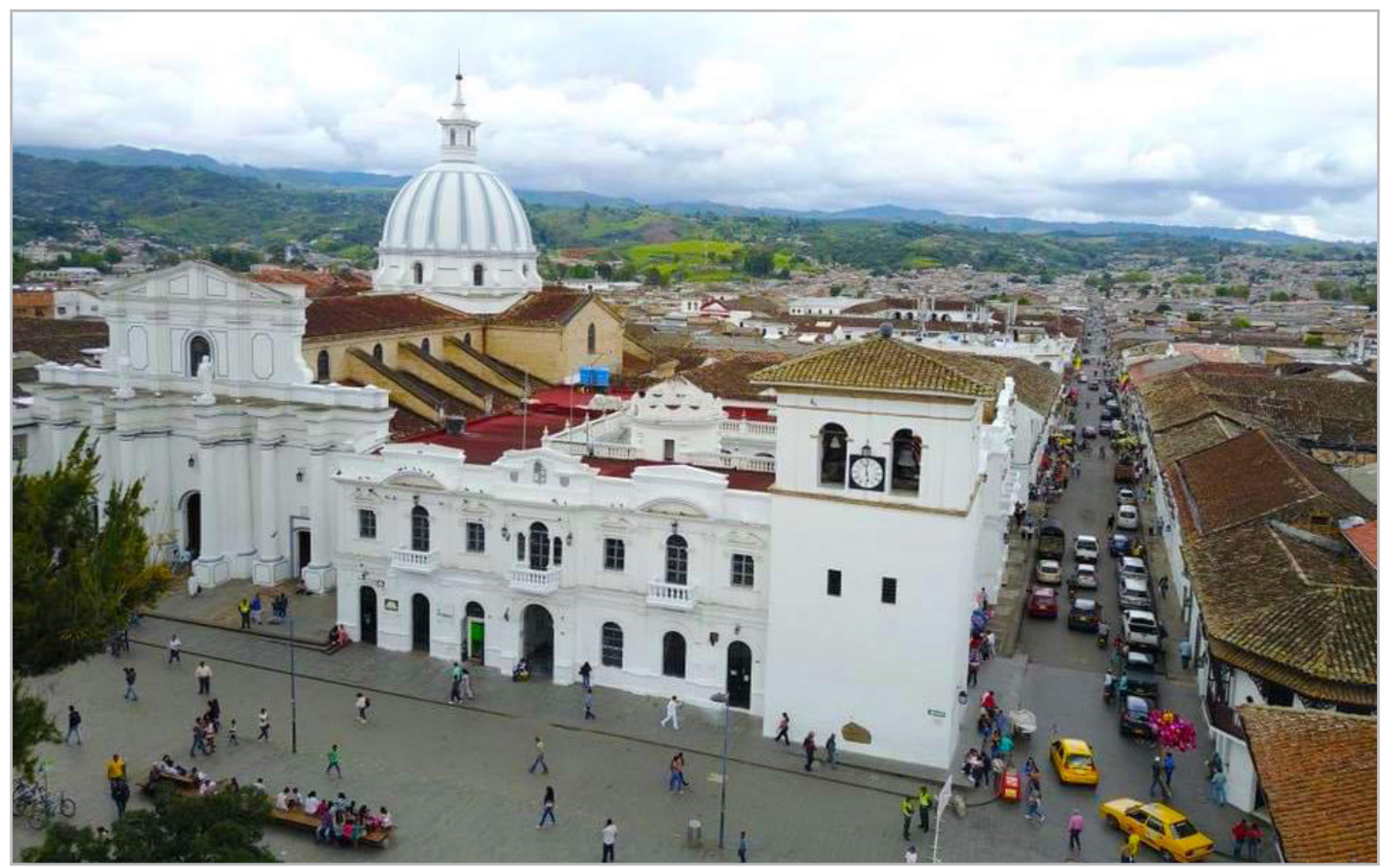

Figura 2.- Vista general de la Parque Caldas en el Sector histórico de la ciudad de Popayán. 
Desde el último terremoto de 1983, se han observado en el centro histórico, cambios en el uso de los materiales durantelas restauraciones, rehabilitaciones oreparaciones de los monumentos, no siempre compatibles con los mismos, igualmente se ha observado un incremento del uso de los espacios públicos y privados y cambios en los usos del suelo que no son favorables para la conservación o que afectan de manera considerable el paisaje urbano [figura 3] y/o la materialidad de los edificios [figura 4].

\section{- Caracterización de la población encuestada}

Las 384 encuestas realizadas a residentes permanentes de Popayán fue acorde a la distribución socio-demográfica de la ciudadanía, donde el $58,3 \%$ de los encuestados son provenientes del resto del país, un $0,8 \%$ son extranjeros, y finalmente un 40,9\% corresponde a población nativa. Un $39,6 \%$ de los encuestados respondió que su nivel educativo eran estudios secundarios, siendo este el porcentaje más alto,
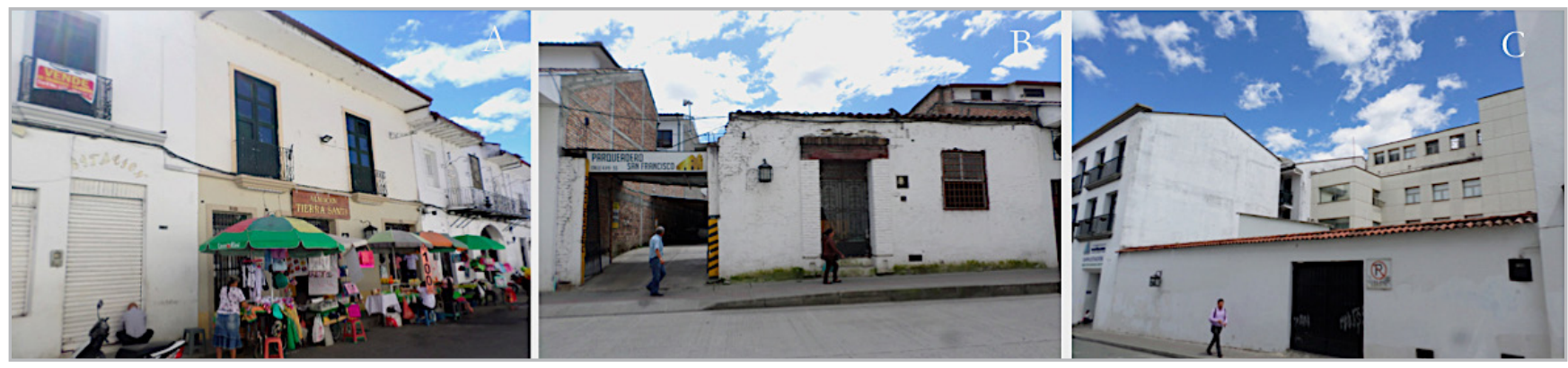

Figura 3.- Alteración del paisaje urbano en el centro histórico A) Ocupación del espacio público, por uso comercial informal, frente a la iglesia de La Encarnación B) Antigua casa convertida en parqueadero sobre la calle 4 frente a la iglesia de San Francisco C) Alteración del perfil urbano por construcción de nuevas edificaciones, edificio sobre la carrera novena, con calle 4.

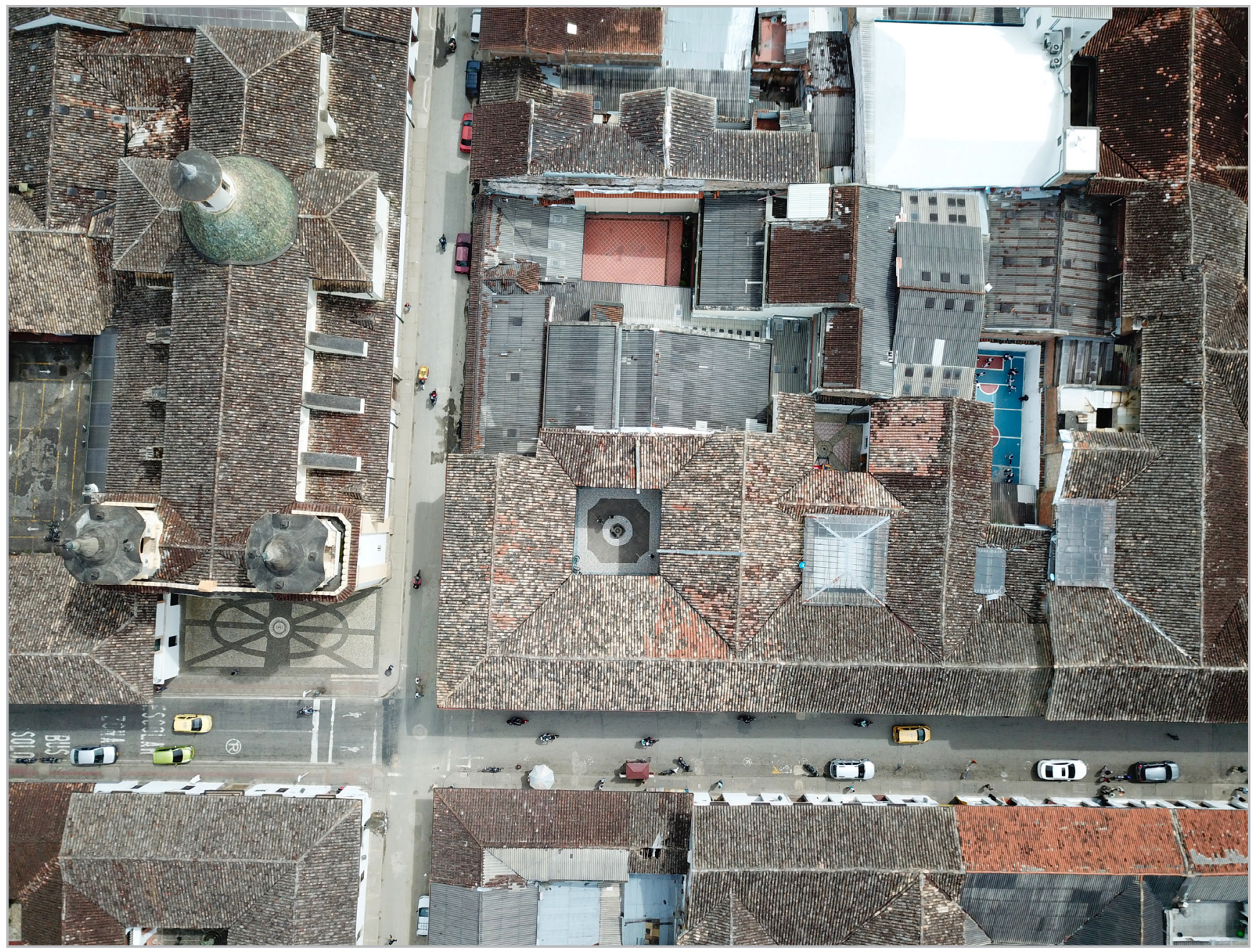

Figura 4.- Ejemplo de la morfología de la manzana contigua a la iglesia de San José en Popayán. Se pueden identificar, en esta vista superior de las cubiertas, cambios en los materiales y técnicas constructivas de las cubiertas de tejas de cerámica. 
Lugar de nacimiento de los encuestados residentes en Popayán

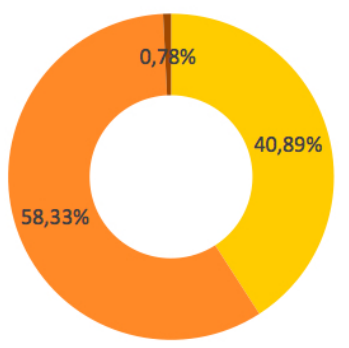

" Popayán
Nivel de estudios alcanzados de los encuestados residentes en Popayán Primaria

" Resto del país

Extranjeros

Secundarios

- Superiores no universitarios

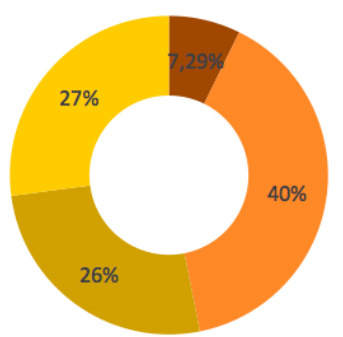

Figura 5.- Caracterización general de la población encuestada

lo que indica que gran parte de la población no ha accedido a la educación superior, universitaria y no universitaria. Para estos casos el $26 \%$ dijo tener estudios superiores no universitarios y un $27,1 \%$ estudios superiores universitarios. Un $7,3 \%$ dijo haber alcanzado sólo estudios de primaria [figura 5].

\section{-Caracterización de la opinión social}

A continuación, se recogen los resultados de las encuestas a partir de cinco aspectos: a) la importancia de la conservación del patrimonio en la ciudad, b) nivel de conocimiento de los monumentos, c) preocupación de los entes gubernamentales por los monumentos de la ciudad, d) visita a los monumentos de la ciudad y e) importancia de los monumentos para la planificación viajes. Estos resultados se analizan desde los rangos de edad, el nivel de estudios y el sitio de origen de los ciudadanos.

a) Opinión de los habitantes de Popayán sobre la importancia de la conservación del patrimonio de su ciudad.

El $79,2 \%$ de los ciudadanos encuestados calificaron en una escala del 1 al 5, que es muy importante la conservación (5); una significativa proporción respecto a las otras opciones, sólo el 0,8\% valoró con uno (1), considerando que es muy poco o nada importante la conservación del patrimonio en la ciudad. Estas respuestas en su mayoría positivas aportan en la generalidad la importancia del patrimonio para los habitantes de Popayán, solo existe un porcentaje bajo de ciudadanos, 2,4\% entre los niveles bajo (2) y muy bajo (1), que manifiestan que no les interesa, o les interesa muy poco, que sea preservado el patrimonio de la ciudad.

Esta opinión varía considerablemente entre los grupos de edad. El rango de edad que calificó con valores más bajos, corresponde a los jóvenes y adultos de temprana edad (18 a 29 años); los grupos de mayor edad fueron más propensos a calificar con valores más altos este apartado. Lo anterior se puede interpretar a partir de una posición de arraigo, apropiación y sentido de comunidad de las personas mayores, que desde su cotidianidad y a través del tiempo han compartido imaginarios y desarrollado conexiones hacia los valores del patrimonio. Por el contrario, los jóvenes probablemente no se involucran de forma directa por lo que no le dan la misma importancia; además, Popayán concentra a una alta población foránea de estudiantes, que permanece en la ciudad, sin necesariamente establecer vínculos o reconocer los valores de su patrimonio. Este valor hace pensar en la necesidad de mejorar la visión de los más jóvenes del patrimonio histórico, independientemente de su origen, especialmente en países no envejecidos, como es el caso de Latinoamérica.

Según el nivel de estudios de los encuestados se observa que el $98,1 \%$ que tienen estudios superiores universitarios le otorgaron una valoración entre 4 y 5 , por el contrario, el $17,9 \%$ de las personas con un nivel de educación primaria fueron los que otorgaron la valoración más baja (1, 2 y 3). Este resultado revela que el nivel de escolaridad y por tanto, el nivel de conocimiento adquirido indica una preocupación y conciencia mayor por el valor del patrimonio y podría ser la clave para fortalecerlo ante las distintas amenazas. Así mismo, nos lleva a pensar sobre la importancia de incluir el concepto de "conservación del patrimonio histórico" en las escuelas primarias como un aspecto curricular.

Según el lugar de origen, el $84,1 \%$ de la población local le otorgó la mayor valoración asignada, al igual que el 75,8\% de la población originaria del resto del país. Esto señala que tanto nativos como allegados dan una gran importancia a los monumentos de la ciudad. Este resultado puede evidenciar la apropiación e identificación a causa de los valores sociales que el patrimonio aporta al imaginario colectivo en el transcurso del tiempo; el fenómeno que crea con las personas no locales que residen en la ciudad puede verse influenciado por la participación y el interés por parte de la población nativa.

b) Opinión de los habitantes de Popayán sobre su nivel de conocimiento de los monumentos de la Ciudad:

El $43 \%$ de los encuestados contestó que el nivel de conocimiento era regular en una escala del 1 al 5. Sólo 
un $3,4 \%$ consideró que su nivel de conocimiento de los monumentos de la ciudad era muy alto, y en el otro extremo, un $6,8 \%$ opinó que era muy bajo. A través de estas respuestas, los ciudadanos expresan que el nivel de conocimiento sobre nuestro patrimonio, no es suficiente, lo que apoya la propuesta sobre la necesidad de incluir el estudio de estos aspectos desde la primaria. Algunos de los entrevistados, no tenían una opinión clara al respecto, favorable o desfavorable, y esto hace mucho más difícil la tarea de conectar de alguna forma los bienes patrimoniales con la sociedad, y mucho más esperar un papel activo de la ciudadanía en cuanto al tema.

En función de los grupos de edad, el que le asignó una mayor calificación (5) a esta pregunta tiene entre 18 y 29 años, y el grupo que dio la valoración más baja (1) tiene más de 60 años. En general las respuestas estuvieron inclinadas a calificarlo como regular (3), siendo los ciudadanos entre los 45 y 59 años los que más insistieron en esta respuesta. Esto indica que el simple hecho de convivir con el patrimonio no es un determinante para conocer el trasfondo detrás de este, y aunque los jóvenes fueron los que expresaron que conocían más el patrimonio, esto no se traduce en que desarrollen un interés particular hacia él, ni un alto grado de apropiación social, lo que se refleja en que no consideran importante su conservación según los resultados recogidos en el apartado anterior.

Según nivel de estudios, todos los grupos educativos concentraron su calificación como regular (3). El grupo con estudios de primaria es el que mejor valoró (5) su nivel de conocimiento, con un $10,7 \%$, tan sólo el $1 \%$ de la población con estudios superiores universitarios piensan que su nivel de conocimiento es muy bueno (5). Las respuestas en esta categoría tuvieron una alta variación, pero se puede señalar que para la ciudadanía no es un punto fuerte el conocer su patrimonio a pesar de tenerlo próximo.

Según el lugar de origen de los ciudadanos, las respuestas en esta categoría varían respecto a la calificación asignada. La población proveniente del resto del país es la que le otorga una mayor valoración (5) con un 4\%, respecto al 2,5\% representado por la población local. Esto podría implicar que los foráneos tienden a percibir que el nivel de conocimiento sobre los monumentos en la ciudad es más alto que lo que consideran los locales. Si bien las diferencias son pequeñas y habría que profundizar en este aspecto.

c) Opinión de los habitantes de Popayán sobre la preocupación de los entes gubernamentales por los monumentos de la ciudad.

El 27,9\% de los encuestados consideran que la preocupación de las organizaciones gubernamentales es muy baja (1), el $33,9 \%$ lo considera bajo (2) y el $29,7 \%$ como regular (3). En contraste, sólo el 0,5\% de los encuestados contestó que el nivel de preocupación es muy alto (5). Esto tiene relación directa con el papel de las instituciones gubernamentales en lo concerniente a la gestión del patrimonio en la ciudad, no sólo en términos de conservación, sino en la activación del sector económico y cultural y en la difusión que se hace del trabajo realizado.

Según los grupos de edad, los ciudadanos entre los 45 y 59 años (con un 41,3\%) opinan que la preocupación de los entes gubernamentales es regular (3). En el caso de la valoración baja (2), es el rango entre los 30 y 44 quien representa un mayor peso porcentual, con un 38\%. En el caso de muy baja preocupación (1), son los ciudadanos de más de 60 años (36,9\%) los que consideran el papel de los políticos y gestores como deficiente.

Según el nivel de estudios, el grupo con estudios superiores universitarios considera mayoritariamente $(47,1 \%)$ como baja la preocupación de los entes gubernamentales por los monumentos. El grupo con estudios de primaria son quienes en mayor proporción calificarían como muy baja (1) la acción de los gestores, seguida de la población estudios superiores no universitarios con un 35,7\% y 33,0\% respectivamente. Tan solo un 1,7\% de los encuestados valora muy positivamente (5) la gestión.

Se evidencia que la opinión generalizada es que existe una deficiente gestión del sector público, tanto de las personas con mayor nivel educativo como de menor nivel educativo, sin embargo, son estas últimas las que peor lo califican, lo que podríamos traducir como una percepción de mayor distanciamiento y exclusión hacia esta población, a la cual probablemente no han llegado las acciones gubernamentales suficientes para involucrarla activamente. Por el contrario, hay que indicar que el centro histórico de Popayán es el más grande conservado en Colombia, su Semana Santa es Patrimonio Inmaterial de la Humanidad y cuenta con un Plan Especial de Manejo y Protección (PEMP) desde el año 2010, por lo que se evidencia la necesidad de medidas de comunicación sociales más efectivas con los diferentes grupos de interés para su participación en los modelos de conservación de la ciudad.

d) Opinión de los habitantes de Popayán sobre visitar los monumentos de su ciudad.

Los encuestados reconocieron en general no visitar los monumentos de la ciudad. El $25.8 \%$ de los encuestados respondieron con un valor 2 , lo que indica que el nivel de visitas es bajo; seguido del $24,2 \%$ con una valoración regular (3) y un $21,9 \%$ con una valoración muy baja (1). Se evidencia que sólo el 9,4\% de los ciudadanos encuestados realiza visitas muy frecuentes, esto tiene implicaciones en la conservación del patrimonio, ya que un bien abandonado progresivamente por la sociedad civil, es más propenso a que desde las instituciones no se garanticen los mecanismos necesarios para mantenerlo en uso y en óptimo estado. Las causas son difíciles de analizar, pero podrían estar en el poco interés o tiempo en el esparcimiento cultural, y las pocas o nulas iniciativas desde los gobiernos nacionales y locales para proporcionar y mantener un rol activo del ciudadano hacia su entorno próximo. 
Según grupos de edad, el grupo de mayores de 60 años y el de 18 a 29 años manifestaron visitar los monumentos de Popayán con mayor frecuencia (5) con porcentajes del $13,8 \%$ y $13,2 \%$ respectivamente. Los ciudadanos entre los 45 y 59 años son los que menos visitas realizan, ya que el $27,2 \%$ contestó que no realizaba visitas a monumentos y el $31,5 \%$ que casi nunca las realizaba.

Según nivel de estudios, los resultados obtenidos muestran que la población con estudios universitarios son los que con mayor frecuencia visitan los monumentos de la ciudad, con un $30,8 \%$ y un $13,5 \%$ para los valores de 4 y 5 respectivamente. Los ciudadanos que menos concurren a los monumentos pertenecen al nivel de estudios de primaria, representando un $42,9 \%$ y $21,4 \%$ con respuestas 1 y 2 respectivamente.

Según el lugar de origen, los resultados reflejan que los ciudadanos que nacieron en Popayán son los que manifestaron visitar de forma más regular los monumentos con las valoraciones 3,4 y 5, con una importante diferencia porcentual respecto a la población foránea. Un 17,2\% de los ciudadanos nacidos en Popayán dijeron que la frecuencia de visitas que hacía a los bienes patrimoniales era muy poco frecuente (1), no obstante, la población no local supera esta cifra con 7,9 puntos porcentuales.

e) Opinión de los habitantes de Popayán sobre la importancia de los monumentos para la planificación de sus viajes.

Los ciudadanos en general no consideraron fundamental este aspecto para desarrollar sus actividades de turismo y ocio. Tan solo un $25,5 \%$ de los encuestados lo valoró como importante (4) y un 19,3\% dijo que era muy importante (5), mientras un $23,7 \%$ lo considera con importancia media (3) y un $14,6 \%$ manifestó que no era nada importante (1).

Según grupos de edad, los mayores de 60 años y los jóvenes y adultos entre 18 y 29 años son los que manifestaron tener más en cuenta los monumentos para la planificación de sus viajes, con resultados totales de $58,4 \%$ y $57,6 \%$ respectivamente para valores de 5 y 4 . Los ciudadanos entre los 45 a 59 años son los que menos tienen en cuenta este aspecto para planificar sus viajes, representando un $45,7 \%$ en total para las valoraciones de 1 y 2 .

Con respecto al nivel de estudios, el grupo de ciudadanos con estudios universitarios es el que más tiene en cuenta este aspecto, un $26,0 \%$ da la valoración más alta (5) mientras un 33,7\% da la valoración (4), lo que significaría que siempre o casi siempre tienen en cuenta los monumentos a la hora de planificar sus actividades de ocio y turismo. Por otro lado, la población que referenció tener solo estudios de primaria, es la que menor valoración le asigna a esta consideración (1 y 2), representando un $42,8 \%$ del total, le siguen las personas con estudios secundarios, entre las que un $16,4 \%$ da la valoración (1), y con porcentajes similares las personas con estudios superiores no universitarios que calificaron también en un $16 \%$ que no es importante (1).

\section{—Evaluación de la opinión social}

La figura 6 recoge un resumen comparativo de los cinco aspectos evaluados mediante las opiniones de los ciudadanos encuestados en Popayán.

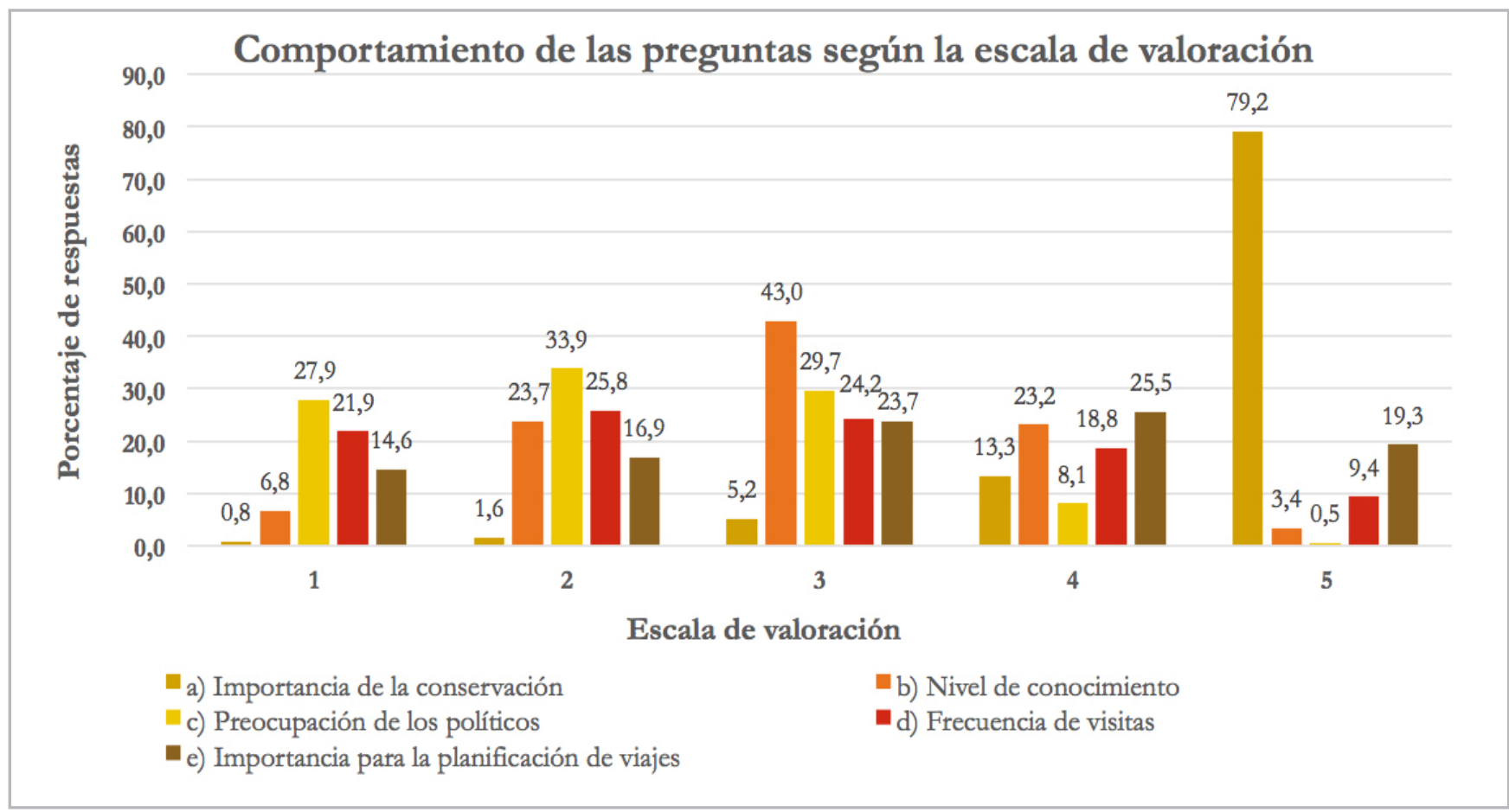

Figura 6.- Opinión social general de los ciudadanos de Popayán (Colombia) recogida en la encuesta sobre el patrimonio 
El resultado del cuestionamiento sorprende por la elevada preocupación que expresan los ciudadanos sobre la importancia de la conservación del patrimonio, el 79,2\% de los encuestados responde con el valor más alto (5), esto independiente del nivel educativo, edad o lugar de origen de los encuestados.

Este dato parece estar en contradicción con las respuestas a las demás preguntas, ya que, aunque existe un alto nivel de preocupación por la conservación del patrimonio histórico en Popayán, sus ciudadanos opinan que su nivel de conocimiento del patrimonio es regular, la preocupación de sus políticos por el patrimonio es muy baja, y gran parte de la población no visita frecuentemente los monumentos ni tiene en cuenta los bienes patrimoniales de forma significativa a la hora de planear sus viajes de turismo y ocio. Esto se podría explicar a partir del concepto de capital social (The Getty Conservation Institute 2013), que tiene que ver con la asociación identitaria, espiritual, ideológica y hasta de tipo histórico y estética que se puede desarrollar. Podría afirmarse que la importancia evidenciada por la ciudadanía puede ser un factor a favor de la conservación del patrimonio, pero dado el comportamiento de las otras variables no ha sido desarrollado en el territorio.

En cuanto al nivel de conocimiento de los bienes patrimoniales, el panorama no es muy positivo, tan solo el $3,4 \%$ lo calificó como muy alto, las respuestas se centraron entre medio y bajo. Esta variable es muy representativa, ya que darle una significación al patrimonio implica conocerlo. La difusión de este tipo de conocimiento no llega a la población en general, entre las posibles causas, encontramos el limitado acceso que tienen al patrimonio, que no motiva a los diferentes grupos de la sociedad, falta de campañas y políticas motivadoras, o la falta de interés de los individuos. Dada la amplia afluencia de personas foráneas en Popayán, es destacable que la población local insista en que el grado de conocimiento no es alto. Es ahí, donde es importante la implementación de estrategias educativas para promover el conocimiento hacia el patrimonio, su uso, su apropiación e incluso su relación como motor del desarrollo social y económico, o de los desarrollos comunitarios. Este estudio deja patente la necesidad de transmitir una visión del patrimonio edificado que vaya más allá de la importancia histórica (García Canclini 1999). Para lograrlo, es imperativo la accesibilidad a los conocimientos, ya que es una condición necesaria para la toma de decisiones, y contribuye a una conservación integrada del patrimonio (Icomos 2015).

En general, la percepción sobre la preocupación de los entes gubernamentales para preservar los monumentos es bastante desalentadora, sólo el 0,5\% de los ciudadanos encuestados la calificarían como muy alta; es decir, en opinión de la población, hay una ausencia de interés o voluntad política para llevar a cabo la labor de protección. Lo cierto es que los ciudadanos no perciben los esfuerzos de los gobiernos y líderes políticos, lo cual ofrece una pista acerca del rol histórico y actual de las instituciones frente al patrimonio; que sean las personas de mayor edad las que consideren que estos actores no tienen como una de sus prioridades el cuidado del patrimonio, demuestra una baja confianza en las instituciones, como gestores del mismo, y por tanto una larga tradición de invisibilidad a la sociedad durante generaciones (Garavito Gonzáles 2006; Caraballo Perichi 2008)

El que los ciudadanos en su mayoría no frecuenten los monumentos de la ciudad es un indicador de la baja interacción que tienen con estos, sólo un 9,4\% respondió que los visitaba de manera habitual; esto indica que, a pesar de convivir desde la cotidianidad, no se establece un vínculo ni reconocimiento con el entorno patrimonial. Una de las posibles causas son las limitadas iniciativas y actividades, que permitan el disfrute, igualmente la apatía o falta de interés hacia el ámbito cultural. Las visitas y la interacción adecuada con el patrimonio promueven un sentido de apropiación y una preocupación por la salvaguarda. Estas medidas pueden prevenir que se caiga en desuso o uso impropio, condiciones que se pueden dar si los espacios no son reconocidos por los ciudadanos para su aprovechamiento.

Por último, es importante anotar que apenas se tienen en cuenta el patrimonio para planificar viajes, tan sólo un 19,3\% aseguró que era muy importante, seguido de un $25,5 \%$ que respondió que era altamente importante. No obstante, para la mayoría no representa un factor determinante o imperativo para escoger el destino de su tiempo de ocio y viajes; esto es coherente con las repuestas asignadas a otros aspectos, como el poco conocimiento sobre el patrimonio y la baja frecuencia de visitas. Esto evidencia aún más el desconocimiento de los ciudadanos acerca de los entornos patrimoniales y a su vez denota poco interés y capacidad de divertirse en buena medida por la ausencia de diversificación y difusión del patrimonio.

\section{- La opinión social como factor agregado en el grado de vulnerabilidad}

El porcentaje de respuestas de cada valor (PRv), calculado con base al número total de respuestas obtenidas para cada uno de los valores (TRv) para la escala de 1 al 5, se representa en la tabla III.

Tabla II.- Método para el cálculo de opiniones por escala de valor

\begin{tabular}{|l|c|c|c|c|c|}
\hline \multicolumn{1}{|c|}{ Escala de valor de cada pregunta } & Valor muy bajo & Valor bajo & Valor medio & Valor alto & Valor muy alto \\
\hline Valor de la opinión social (V) & 1 & 2 & 3 & 4 & 5 \\
\hline Total respuestas de cada valor (TRv) & 276 & 391 & 483 & 341 & 429 \\
\hline Porcentaje de respuestas de cada valor (PRv) & $14.40 \%$ & $20.40 \%$ & $25.20 \%$ & $17.80 \%$ & $22.30 \%$ \\
\hline
\end{tabular}




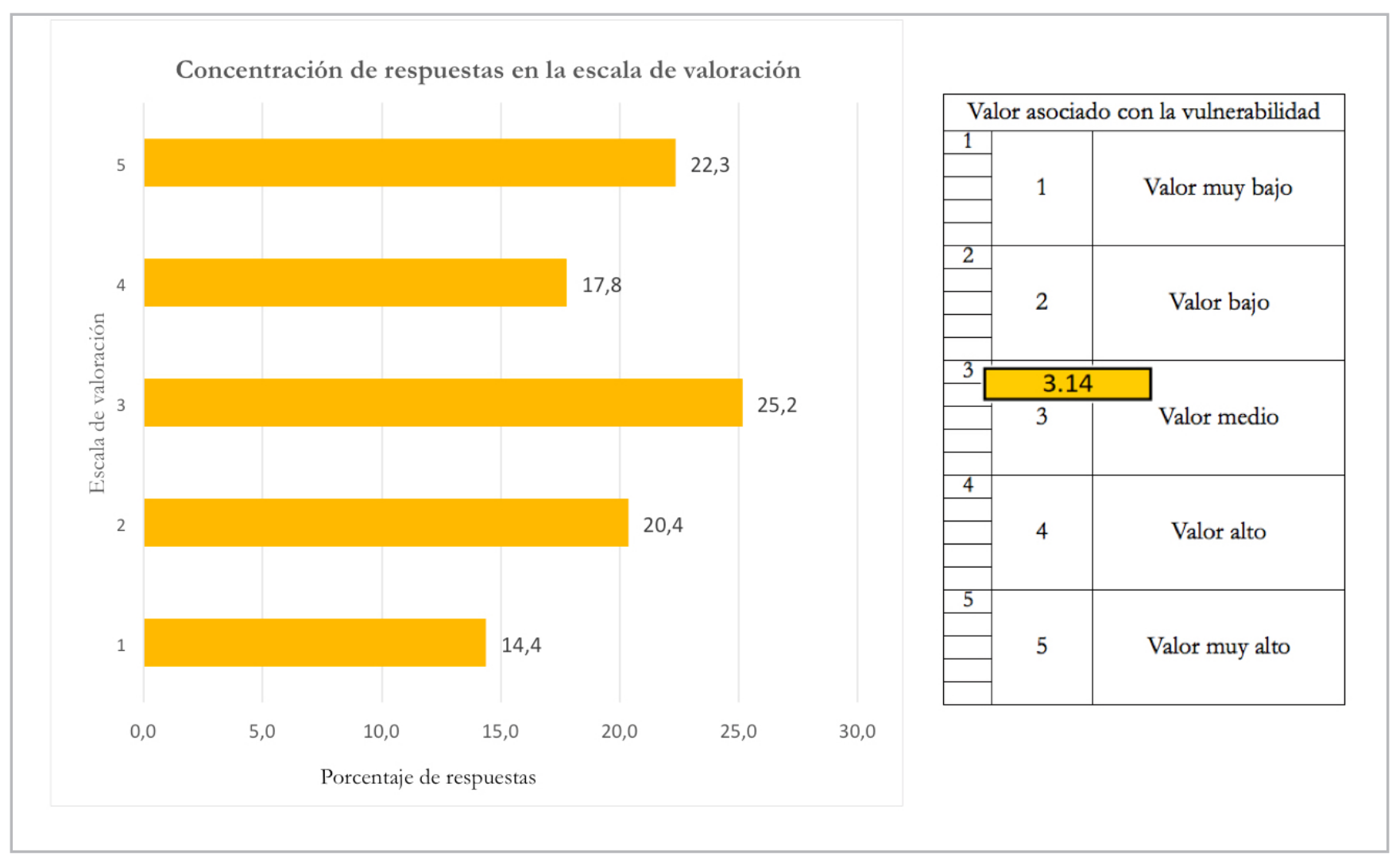

Figura 7.- Grado de vulnerabilidad del patrimonio de Popayán según la opinión social de sus habitantes

A partir de estos datos se ha calculado el grado de vulnerabilidad en función de las opiniones sociales, aplicando la ecuación recogida en la metodología. El grado de vulnerabilidad del patrimonio de Popayán asociada a la opinión de los encuestados, se puede estimar como medio, correspondiente a un valor de 3.14, en una escala de medida de 1 a 5, siendo (1), muy vulnerable y (5) muy poco vulnerable. [figura 7]

En Popayán, como en la mayoría de ciudades hispanoamericanas, están latentes las dinámicas de orden global, que pueden afectar a la opinión de la sociedad frente al patrimonio, como el crecimiento demográfico y aumento de la pobreza, el consumismo, entendido como la visión a corto plazo, la moda global promocionada por los medios de comunicación, la cultura global que discrimina las diversidades culturales, la falta de recursos profesionales para atender técnicamente el patrimonio y la ausencia de conocimiento esencial sobre los monumentos, entre las principales causas enumeradas en el informe de ICOMOS (ICOMOS 2000) sobre monumentos y sitios en peligro. A estas causas, se suman otras de orden nacional o regional que están relacionadas con los problemas de índole colectivo como la fragmentación social, la diversidad en la composición cultural de la población actual, producto de las migraciones y el desplazamiento forzado del campo a las ciudades; además, la falta de alternativas de inversión pública, para suplir la necesidades básicas como la vivienda y la educación, ocasionando cambios socioeconómicos y llevando progresivamente a la pérdida de la identidad cultural.

Las implicaciones sobre los bienes patrimoniales ya se perciben en el centro histórico de Popayán, y pueden estar asociadas a esta baja-media valoración social del patrimonio. Entre esas implicaciones hay actuaciones de la población, que sobrepasan la existencia de la normativa, como son los usos impropios del suelo, la ocupación informal del espacio público con fines lucrativos, o la construcción de edificaciones ilegales afectando el paisaje urbano y la contaminación visual. Estas amenazas al patrimonio histórico pueden tender a incrementarse en el futuro de forma progresiva, en especial por combinación con otros factores, como la presión urbanística, bajo políticas de gestores poco sensibilizados o preocupados por la conservación del Patrimonio. Por otro lado, la falta de conocimiento sobre el valor del patrimonio, puede afectar a proliferación de intervenciones u obras de mantenimiento inadecuadas, que sumada a la carencia de conocimientos técnicos o falta de documentación, puede llevar a actuaciones que desfiguren la materialidad del patrimonio. Además, la ausencia de reconocimiento de los valores tangibles e intangibles del patrimonio puede favorecer el robo, el vandalismo, el abandono o los usos no compatibles; que a la larga ocasionan el debilitamiento físico del monumento, su pérdida de significación cultural, la ruina o incluso su desaparición [figura 8]. 

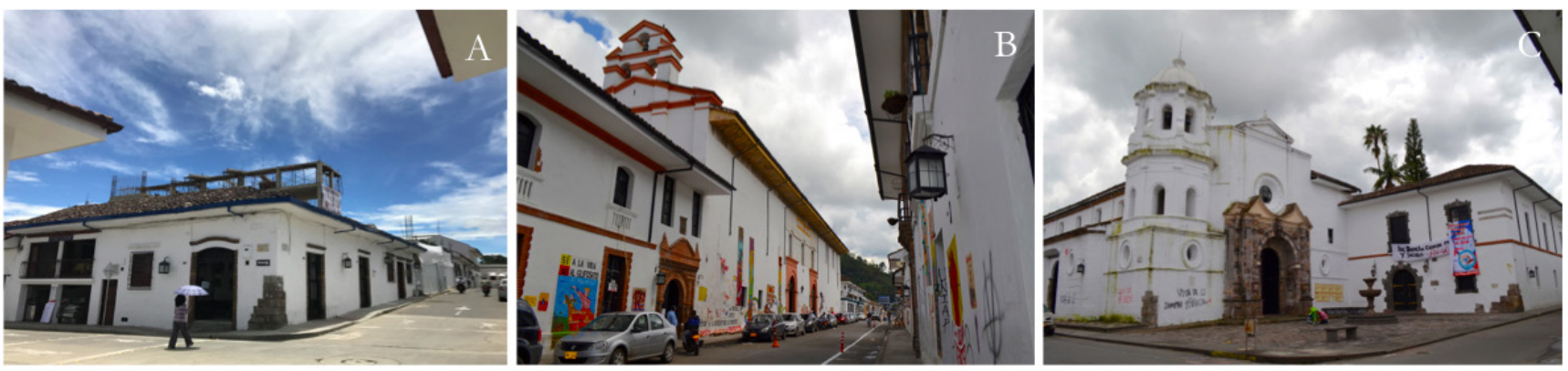

Figura 8.- Ejemplos de alteración en la conservación del patrimonio de Popayán. A) Construcción de edificio modificando los perfiles urbanos ubicado en la Carrera 10 con calle 4 en el sector histórico. B) Grafitis como muestra de actos vandálicos afectando el paisaje urbano en la iglesia de El Carmen. C) Contaminación visual y vandalismo por grafiti en la Iglesia de santo Domingo.

\section{Conclusión}

La metodología desarrollada en este trabajo puede contribuir a valorar la vulnerabilidad del patrimonio, desde la óptica de las comunidades allegadas. Los resultados ponen de manifiesto la importancia de evaluar el comportamiento social que puede afectar de manera directa al patrimonio, como es el caso de los actos vandálicos o la presión urbanística, o de manera indirecta por las corrientes de orden global y/o conflictos de intereses. En este sentido, estimar la conciencia y principales preocupaciones de los ciudadanos ante la preservación del patrimonio podría medir el nivel de sensibilidad social acerca de la importancia que tiene su conservación, e identificar las necesidades actuales de la población y las medidas para fortalecer la conservación como factor de identidad y desarrollo sostenible.

Los resultados obtenidos en esta investigación son útiles para el planteamiento de estrategias basadas en la colaboración y participación activa de los ciudadanos, donde tengan la posibilidad de comprender, valorar y proteger el patrimonio. El análisis realizado se basa en un componente subjetivo y cualitativo, relacionado con la percepción de los habitantes de Popayán, del patrimonio de su ciudad, y por tanto hay una incertidumbre asociada a la medición y evaluación, que debe ser analizada en futuros estudios. En este sentido, la relación del ciudadano con su patrimonio implica múltiples escalas de percepción basadas en sus necesidades a nivel individual y comunitario, sin embargo, pueden identificarse patrones de valoración discernibles y cuantificables vinculados a causas frecuentes de deterioro o de conservación del patrimonio edificado, por lo que la metodología planteada se puede usar para campañas que impliquen formas de actualización del patrimonio que tengan como fin su conservación.

Este estudio pone de manifiesto la necesitad de la coparticipación de distintos actores gubernamentales y no gubernamentales, que puedan involucrarse en la gestión de los bienes de interés cultural, la estimación e implementación de políticas de actualización del valor del patrimonio para elevar el grado de significación de las comunidades más próximas al él, y a su vez la creación de todo un escenario donde se promuevan los recursos patrimoniales.

\section{Agradecimientos}

Esta investigación se basa en la Metodología desarrollada por dos Proyectos: ART-RISK, un proyecto RETOS del Ministerio de Economía y Competitividad y Fondo Europeo de Desarrollo Regional (FEDER), (código: BIA2015-64878-R (MINECO / FEDER, UE)) y el proyecto UPO-03 de la consejería de Fomento, Infraestructura y Ordenación del Territorio de la Junta de Andalucía (España). Pilar Ortiz agradece la beca Salvador Madariaga del Ministerio de Educación, Cultura y Deporte del Gobierno de España (PRX16/00226) para la realización de una estancia en la Universidad de Oxford (UK), durante la cual se trabajó en el diseño de las encuestas utilizadas en esta investigación. Rocío Ortiz agradece su estancia en la Universidad de Lisboa (Portugal), durante la cual se trabajó en el diseño de las encuestas utilizadas en esta investigación.

\section{Referencias}

ALCALDÍA DE POPAYÁN. (2015). Plan de Movilidad para el municipio de Popayán. Informe 3: diagnóstico parte, $1^{\circ} \mathrm{ed}$., Popayán: Movilidad Futura.

BENÍTEZ, J., TIRADO-HERNÁNDEZ, A., ORTIZ, R,. MORENO, M., ORTIZ, P., TURBAY, I., GARRIDO-VIZUETE, MA. (2020). "Social opinion of monuments vulnerability in historical cities". Science and Digital Technology for Cultural Heritage, Ortiz, P (coord.) Sevilla: Taylor \& Francis Group, 221-224. https://doi. org/10.1201/9780429345470-41

CARABALLO PERICHI, C. (2008). "El patrimonio cultural y los nuevos criterios de intervención. La participación de los actores sociales" Palapa. Revista de Investigación Científica en Arquitectura/ Journal of Scientific Research in Architecture, 3:41-49.

DANE (2019). Resultados Censo Nacional de Población y Vivienda 


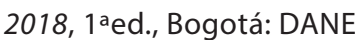

DIAZ FUENTES, D. A. (2017). “Un método simplificado para evaluar el riesgo sísmico y priorizar la atención de los bienes culturales inmuebles: el caso de Chile", Intervención, 8:46-62. https://doi.org/10.30763/Intervencion.2017.15.173

GARAVITO GONZÁLES, L. (2006). “El origen del patrimonio como política pública en Colombia, y su relevancia para la interpretación de los vínculos entre cultura y naturaleza". Opera, 6:169-187.

GARCÍA CANCLINI, N. (1999). "Los usos sociales del patrimonio cultural", Patrimonio Etnológico. Nuevas perspectivas de estudio, 1: 16-33.

GARRÉ, F. (2001). "Patrimonio arquitectónico urbano, preservación y rescate: bases conceptuales e instrumentos de salvaguarda", Conserva. 5:5-21.

ICOMOS. (2015). Documentos de icomos, $1^{\text {a }}$ ed., Santiago de Chile: J. de N. Concha, Ed.

ICOMOS. (1999). Carta de Burra, $1^{\text {a }}$ ed., Sidney: ICOMOS Australia.

ICOMOS. "Tendencias, Amenazas Y Riesgos" en Informe Mundial 2000 de ICOMOS sobre monumentos y sitios en peligro. https://www.icomos.org/risk/world report/2000/ trends spa.htm?fbclid=IwAR2 waaCDSNTXRJD1STcIZjR ml b6c1xmhU mT6ygSi42cMUPmZ1m197dLDY. [consulta: 3/2/2020].

LLULL PEÑALBA, J. (2005). "Evolución del concepto y de la significación social del patrimonio cultural", Arte, Individuo y Sociedad, 17:175-204. https://dx.doi.org/10.5209/ARIS

MANZINI, L. (2011). "El significado cultural del patrimonio", Estudios del Patrimonio Cultural, 6:27-42.

MINISTERIO DE AMBIENTE VIVIENDA Y DESARROLLO TERRITORIAL. (2006). Serie Población, Ordenamiento y Desarrollo. Guía metodológica 1: Elementos Poblacionales Basicos para la Planeación, 1a ed., Bogotá: Dirección de desarrollo territorial.

ORTIZ, R., ORTIZ, P. (2016). "Vulnerability Index: A New Approach for Preventive Conservation of Monuments", International Journal of Architectural Heritage, 10:1078-1100. https://doi.org/10.1080/15583058.2016.1186758

PRIETO, A.J., TURBAY, I., ORTIZ, R., CHÁVEZ, M.J., MACÍASBERNAL, J.M., ORTIZ, P. (2020). “A Fuzzy Logic Approach to Preventive Conservation of Cultural Heritage Churches in Popayán, Colombia". International Journal of Architectural Heritage, 0:1-20. https://doi.org/10.1080/15583058.2020.173 $\underline{7892}$

THE GETTY CONSERVATION INSTITUTE. (2013). Assesing the values of cultural heritage, $1^{\text {a }}$ ed., Los Angeles: De la Torre, M

TURBAY I., ORTIZ P., ORTIZ, R. (2019). "Assessment of Vulnerability Index Applied to Churches of 18th Century in the Historic Center of Popayan (Colombia)". En Resilience and sustainability of cities in hazardous environments, Dobran, F. (coord.) New York, 26-30.

UNESCO. "Tesauro de la UNESCO" en UNESCO. http:// vocabularies.unesco.org/browser/thesaurus/en/. [4/1/2020].

\section{Autor/es}

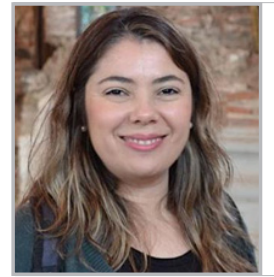

\section{María Isabel Turbay Varona}

isaturbay@hotmail.com

Facultad de Arquitectura, Fundación Universitaria de Popayán, Popayán, Colombia

Arquitecta, Master en Diagnóstico del estado de conservación del Patrimonio Histórico, Candidata a Doctora en Historia y estudios Humanísticos, Europa, América, arte y lenguas, en la línea de Tutela, Protección, Gestión y Puesta en Valor del Patrimonio Histórico, de la Universidad Pablo de Olavide de Sevilla. Profesora en la Facultad de Arquitectura de la Fundación Universitaria de Popayán, (Colombia), donde actualmente es Codirectora del grupo de investigación, GREP, Grupo de Estudios del Paisaje. Su experiencia radica en investigación de la vulnerabilidad y riesgos en el Patrimonio arquitectónico, Valoración social del patrimonio y arqueología de la arquitectura. Ha sido docente en la Facultad de Ingeniería Civil de la Universidad del Cauca, en la Facultad de Arte y Diseño del Colegio Mayor del Cauca y en la Facultad de Artes de la Universidad Antonio Nariño, (Colombia). Ha realizado estancias de investigación en instituciones como el Centro de Investigaciones regionales de Mesoamérica CIRMA en La Antigua (Guatemala), en el laboratorio Sanit-ARTE de la Facultad de Ciencias Experimentales de la Universidad Pablo de Olavide de Sevilla y en el Museo Histórico Municipal de Écija (España) y participó en la III Campaña de Excavación Arqueológica en el Palacio de Villa Adriana, Roma (Italia).

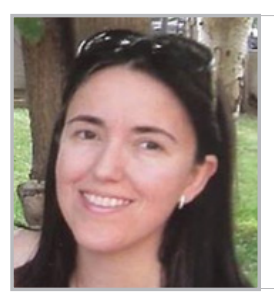

\section{Rocío Ortiz}

rortcal@upo.es

Departamento de Sistemas Físicos, Químicos y Naturales, Universidad Pablo de Olavide, Sevilla, España

Licenciada en Arquitectura en 2001, Máster en Protección del Patrimonio Histórico con un Proyecto enfocado en el estudio de Marchena en 2012. En 2014 Obtuvo el título de Doctora con mención europea en Universidad Pablo de Olavide (Sevilla). Actualmente es profesora en la UPO, donde desarrolla su actividad investigadora en torno a la Conservación Preventiva y el estudio de riesgos y vulnerabilidad del Patrimonio Histórico. 
La Dra. Ortiz es directora del Máster de Diagnóstico del Estado de Conservación del Patrimonio Histórico y es responsable del Área de Cristalografía y Mineralogía de la misma universidad. La Dr. Ortiz es experta en el diagnóstico del Patrimonio Histórico, administración, e-learning, y sus investigaciones científicas aparecen publicadas en 30 libros, actas de congresos, y revistas especializadas. Sus proyectos de investigación han sido financiados por la Unión Europea, gobiernos nacional y autonómico, y organizaciones privadas. Los intereses de la Dr. Ortiz se centran en los estudios de riesgo y vulnerabilidad del Patrimonio Cultural, el empleo de información georreferenciada para el estudio del Patrimonio Cultural, materiales de construcción, planes de ordenación urbana y peligros e innovación en las enseñanzas de ciencias con especial énfasis en el diagnóstico del Patrimonio Histórico.

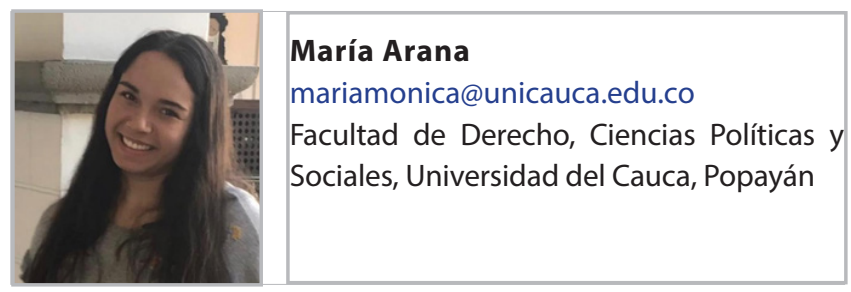

Estudiante de Ciencia Política de la Universidad del Cauca. Se desempeña como coordinadora general del semillero de investigación Ciudad, Universidad y Juventud, en el cual desarrolla investigaciones en las líneas de comunicación política y ciudad, con particular interés en la cohesión de las instituciones públicas y la sociedad civil en la gestión de las ciudades. Ha publicado textos en la revista universitaria Comarca de la Universidad del Cauca. Realizó un voluntariado en Houston, Texas.

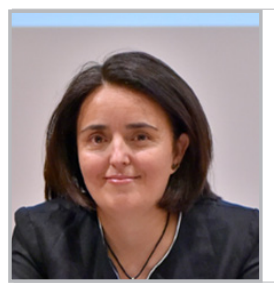

\author{
Pilar Ortiz \\ mportcal@upo.es \\ Departamento de Sistemas Físicos, \\ Químicos y Naturales, Universidad Pablo de \\ Olavide, Sevilla, España
}

La experiencia investigadora y docente de la Dra. Ortiz se ha enfocado en el diagnóstico, preservación y conservación del Patrimonio Cultural. Para su desarrollo ha colaborado con museos y otras instituciones culturales como el IVCR+i, IAPH, Museo de Cádiz, Museo de Écija, etc. Además, ha desarrollado de actividad en diferentes países: Reino Unido, Bélgica, Italia, Rumanía, Cuba, Colombia, Panamá, etc. Su producción científica puede ser resumida en 38 publicaciones en revistas indexadas; 50 libros y capítulos de libros, participación en más de 25 congresos internacionales, chairman y miembro de comités científicos en numerosos congresos, etc. Ha trabajado como profesora asociada en la Universidad de Amberes (Bélgica), como profesora invitada en la Universidad de Oxford (Reino Unido) y como investigadora invitada en ENEA (Italia). La Dra. Ortiz ha participado en 24 proyectos nacionales e internacionales, contratos y convenios y ha sido la investigadora principal de más de 16 de estos proyectos, destacando un Proyecto de Excelencia de la Junta de Andalucía, un proyecto RETOS del Gobierno de España, un Proyecto de Cooperación Internacional de la Junta de Andalucía y un Proyecto Internacional Erasmus+. Ha iniciado y liderado importantes líneas de investigación como la aplicación de técnicas láser para el diagnóstico in situ de Bienes Culturales, el desarrollo de técnicas no destructivas para el estudio del Patrimonio Histórico, o la aplicación de mapas de riesgos, índices de vulnerabilidad e inteligencia artificial para la preservación del Patrimonio Cultural. La Dra. Ortiz es miembro del Campus de Excelencia Internacional de la Universidad de Jaén en Patrimonio Cultural y Natural, del grupo de trabajo "Cambio" en el Campus Internacional de Excelencia de la universidad Pablo de Olavide (UPO), del Consejo Andaluz de Patrimonio Histórico y decana de la Facultad de Ciencias Experimentales de la UPO. 